Pacific Journal of Mathematic 


\title{
THE ASYMPTOTIC DISTRIBUTION OF THE EIGENVALUES FOR A CLASS OF MARKOV OPERATORS
}

\author{
R. M. Blumenthal and R. K. Getoor
}

1. Introduction. This paper is an extension of the preceding paper "Markov Operators and their Associated Semi-groups" (hereafter referred to as MO) by R. K. Getoor. Throughout this paper we will retain the terminology, notations, and all the assumptions of $\S 2$ of MO. Let $G$ be an open subset of $X$ with $m(\partial G)=0$ and suppose that for each $t>0$, $f(t, x, y)$ is in $L_{2}(G \times G, m \times m)$. This is condition $(K)$ in $\S 6$ of MO. Assume further that $f(t, x, y)=f(t, y, x)$ for all $t, x, y$ and, for simplicity, that $f(t, x, y)>0$ for all $t, x, y$. These assumptions will be retained throughout this paper. It is proved in $\S 6$ of MO that under these conditions there is a non-decreasing sequence $\left\{\lambda_{j}\right\}$ of non-negative numbers tending to infinity and a complete orthonormal set $\left\{\varphi_{j}\right\}$ in $L_{2}(G, m)$ such that the series

$$
\sum_{j=1}^{\infty} e^{-\lambda} j^{t} \varphi_{j}(x) \varphi_{j}(y)
$$

converges absolutely. It is further proved that if $k(t, x, y)$ denotes this sum (with $k(t, x, y)=0$ if $x$ or $y$ is not in $G$ ) then $K(V, G ; t, x, A)=$ $\int_{A} k(t, x, y) d m(y)$ for all $t>0, x$ in $G, A$ in $\mathscr{P}(X)$.

Intuitively one can think of $k$ as the transition density of a Markov process that is obtained from $x(t)$ by "killing" $x(t)$ at the boundary of $G$ and upon which a "local death rate" $V(x)$ is imposed. From this interpretation one would expect $k(t, x, y)$ to behave, in some sense, like $f(t, x, y)$ at least for small $t$ and $y$ close enough to $x$, provided $x$ is in $G$ and $V$ is bounded. In the terminology of Kac [4] "the boundary and death rate aren't felt for small $t$ '. In $\S 2$ we make this statement precise by proving that if $V$ is bounded and a certain regularity condition is imposed on $f$, then for all $x$ in $G, k(t, x, y) f(t, x, y)^{-1} \rightarrow 1$ as $t \rightarrow 0$ for almost all $y$ in a suitable neighborhood of $x$ (Theorem 2.1). From this we are then able to show the somewhat surprising fact that $k(t, x, x) f(t, x, x)^{-1} \rightarrow 1$ as $t \rightarrow 0$ for all $x$ in $G$ (Theorem 2.2). Using these facts we derive the asymptotic distribution of the eigenvalues $\left\{\lambda_{j}\right\}$ for a wide class of processes (Theorem 2.3). In $\S 3$ we apply this theory to the symmetric stable processes on the real line and to the OrnsteinUhlenbeck processes.

Received July 8, 1958. The first author was supported, in part, by the Office of Naval Research. 
2. The main theorems. Let $\left\{\left(\ell^{\prime}, \ell^{\prime}\right), P_{x}\right)_{j x \in X}$ be the probability spaces constructed in $\S 2$ of $\mathrm{MO}$. Let $G(t)=\{x(\cdot): x(\tau) \in \bar{G} ; 0 \leqq \tau \leqq t\}$ and let $H(t)$ be the complement in of $G(t)$, that is

$$
H(t)=\{x(\cdot): x(\tau) \notin \bar{G} \text { for some } \tau \leqq t\} .
$$

It was shown in MO that $G(t)$, and hence $H(t)$, are in $\mathscr{S}(t)$.

From the definition of $k$ above and the orthonormality of $\left\{\mathcal{P}_{j}\right\}$ it follows that

$$
k(t+s, x, y)=\int k(t, x, z) k(s, z, y) d m(z)
$$

for all $t, s, x, y$, and that $k(t, x, y)=k(t, y, x)$ for all $t, x, y$. Since $K(V, G ; t, x, A) \leqq p(t, x, A)$ it follows that for each $t$ and $x, 0 \leqq k(t, x, y) \leqq$ $f(t, x, y)$ a.e. $(m)$, and from (2.1) and the symmetry of $k$ and $f$ it follows that these inequalities hold for all $y$. From now on we will assume that $V$ is bounded on $\bar{G}$. In this case we have, for $x$ in $G$, $e^{-M t} K(0, G ; t, x, A) \leqq K(V, G ; t, x, A) \leqq K(0, G ; t, x, A)$ where $M$ is any upper bound of $V$ on $\bar{G}$. If, for the moment, we let $k$ and $k^{\prime}$ denote the densities of $K(V, G ; t, x, A)$ and $K(0, G ; t, x, A)$ respectively, defined by the corresponding series above, then for each $t$ and $x$

$$
e^{-M t} k^{\prime}(t, x, y) \leqq k(t, x, y) \leqq k^{\prime}(t, x, y)
$$

and since $k$ and $k^{\prime}$ each satisfy (2.1) and are symmetric these inequalities hold for all $y$.

In the remainder of this section we will assume that the density $f$ satisfies the following condition:

(D) for every compact set $A$ and every $\eta>0$ there are numbers $t_{0}>0$ and $M>0$ such that $f(\sigma, x, y) f(t, x, z)^{-1} \leqq M$ for all $\sigma \leqq t \leqq t_{0}, x$ in $A, y$ and $z$ in $X$ with $\rho(x, y) \geqq \eta, \rho(x, z)<\eta$. ( $\rho$ is the metric on $X$.)

In the applications, where $X$ is the real line and $\rho$ is the usual metric we will verify this condition for certain familiar process densities.

TheOREm 2.1. For each $x$ in $G$ there is an open neighborhood $U \subset G$ of $x$ such that $k(t, x, y) f(t, x, y)^{-1} \rightarrow 1$ as $t \rightarrow 0$ for almost all $y$ in $U$. (Note that an assumption of $\mathrm{MO}$ is that the support of $m$ is $X$ and hence $m(U)>0$ whenever $U$ is open and non-empty.)

Proof. In view of (2.2) and the remark following it we may assume $V \equiv 0$. Let $q(t, x, y)=f(t, x, y)-k(t, x, y)$. Then

$$
\begin{aligned}
\int_{A} q(t, x, y) d m(y) & =P_{x}[H(t) \cap\{x(\cdot): x(t) \in A\}] \\
& =Q(G ; t, x, A) .
\end{aligned}
$$


Fix $x$ in $G$ and let $S_{z}(x)$ be an open $\varepsilon$-neighborhood of $x$ which is wholly contained in $G$. Let $\delta>0$, be such that $4 \delta<\varepsilon$ and $S_{2 \delta}(x)$ has compact closure. Now if $\left\{x_{k}\right\}$ is a countable dense subset of $X$ then for every $r_{0} \geqq 1\left\{S_{1 / r}\left(x_{k}\right) ; r \geqq r_{0}, k \geqq 1\right\}$ is a countable family of sets which generates $T(X)$. Thus we can construct a sequence $\left\{. / l_{n}\right\}$ of finite partitions of $X$ into $(X)$ sets such that for every $n, \mathscr{C}_{n+1}$ is a refinement of $\|_{n}, P(X)$ is generated by the sets in these partitions, and any set in any of these partitions which intersects $S_{\delta}(x)$ is contained in $S_{2}(x)$. Since $Q(G ; t, x, \cdot)$ is absolutely continuous with respect to $p(t, x, \cdot)$ and since $q(t, x, y) f(t, x, y)^{-1}$ is the derivative of $Q$ with respect to $p$, it follows from known theorems on derivatives (see [2], pp. 343-344) that for almost all $y$ in the sense of $p(t, x, \cdot)$ and hence for almost all $y$ in the sense of $m$

$$
\lim _{n \rightarrow \infty} \frac{Q\left(G ; t, x, B_{n}\right)}{p\left(t, x, B_{n}\right)}=\frac{q(t, x, y)}{f(t, x, y)}
$$

where $B_{n}$ denotes that element of $\mathscr{C}_{n}$ which contains $y$. (The quotients on the left are taken to be 0 whenever the denominator vanishes.)

Given any $t>0$ let $\left\{T_{k}\right\}\left(T_{k}=\left\{t_{k 1}<\cdots<t_{k k}\right\}\right)$ with $t_{k 1}=0$ and $t_{k k}=t$ be an increasing sequence of subsets of $[0, t]$ becoming dense in $[0, t]$ as $k \rightarrow \infty$. Let

$$
\Lambda_{k j}=\left\{x(\cdot): x\left(t_{k j}\right) \notin \bar{G}, x\left(t_{k l}\right) \in \bar{G} ; l=1, \cdots, j-1\right\}
$$

and let $\Lambda_{k}=\bigcup_{j=1}^{k} \Lambda_{k j}$. For each $k$ the $\Lambda_{k j}$ 's are disjoint and $\Lambda_{k} \subset \Lambda_{k+1}$. Moreover $\bigcup_{k=1}^{\infty} \Lambda_{k}=H(t)$ so that for any $B \in \mathscr{P}(X)$ we have

$$
Q(G, t, x, B)=\lim _{k \rightarrow \infty} \sum_{j=1}^{k} P_{x}\left[\Lambda_{k j} \cap\{x(\cdot): x(t) \in B\}\right] .
$$

For each $x$ in $G$ and $A$ in $\mathscr{P}(X)$ define $\left(p(0, x, A)=I_{A}(x)\right)$

$$
\mu_{k j}(x, A)=\int_{\bar{G}} \cdots \int_{\bar{G}} p\left(t_{k 1}, x, d x_{1}\right) \cdots p\left(t_{k j}-t_{k(j-1)}, x_{j-1}, A\right) .
$$

Then $\mu_{k j}(x, X-\bar{G})=P_{x}\left[\Lambda_{k j}\right]$ and

$$
\begin{aligned}
P_{x}\left[\Lambda_{k j} \cap\right. & \{x(\cdot): x(t) \in B\}] \\
& =\int_{x-\bar{a}} \mu_{k j}\left(x, d x_{j}\right) \int_{B} f\left(t-t_{k j}, x_{j}, y\right) d m(y)
\end{aligned}
$$

provided $t_{k j}<t$. On the other hand if $t_{k j}=t$ and $B \subset G$ the left side of this last equation is 0 , so for convenience we define the right side to be 0 in this case. If $B_{n}$ is in $\mathscr{C}_{n}$ and $B_{n} \subset G$ 


$$
\begin{aligned}
& \frac{Q\left(G ; t, x, B_{n}\right)}{p\left(t, x, \bar{B}_{n}\right)} \\
& =\lim _{k \rightarrow \infty} \frac{\sum_{j=1}^{k} \int_{X-\bar{G}} \mu_{k j}\left(x, d x_{j}\right) \int_{B_{n}} f\left(t-t_{k j}, x_{j}, z\right) d m(z)}{\int_{B_{n}} f(t, x, z) d m(z)} .
\end{aligned}
$$

We wish to apply condition (D) with $A=\overline{S_{2 \delta}(x)}$ and $\eta=2 \delta$. Let $y$ be in $S .(x)$ and let $B_{n}$ be that element of $\mathscr{C}_{n}$ which contains $y$. By construction $B_{n} \subset S_{2 \delta}(x)$ so if $z$ is in $B_{n}$ and $x_{j}$ is in $X-\bar{G}$ then $\rho(x, z)<2 \delta$ and $\rho\left(x_{j}, z\right)>2 \delta$. Thus for sufficiently small $t$ the right side of (2.4) does not exceed $M \cdot P_{x}[H(t)]$. This estimate depends on $B_{n}$ only through the fact that $B_{n} \subset S_{2 \delta}(x)$ so combining this with (2.3) we see that

$$
q(t, x, y) f(t, x, y)^{-1} \leqq M P_{x}[H(t)]
$$

for almost all $y$ in $S_{\delta}(x)$ provided $t$ is small enough (how small not depending on $y$ ). Then for almost all $y$ in $S_{\delta}(x)$ we have

$$
1 \geqq \frac{k(t, x, y)}{f(t, x, y)} \geqq 1-M P_{x}[H(t)] .
$$

By the right continuity of the paths $P_{x}[H(t)] \rightarrow 0$ as $t \rightarrow 0$ and so if we take $U=S_{\delta}(x)$ the proof of Theorem 2.1 is complete.

Theorem 2.2. For all $x$ in $G, k(t, x, x) f(t, x, x)^{-1} \rightarrow 1$ as $t \rightarrow 0$.

Proof. If $x$ and $\delta$ are as in the preceding proof then

$$
\begin{gathered}
1 \geqq \frac{k(2 t, x, x)}{f(2 t, x, x)}=\frac{\int k(t, x, y) k(t, y, x) d m(y)}{\int f(t, x, y) f(t, y, x) d m(y)} \\
\geqq \frac{\left(\int_{s_{\delta}(x)} k^{2}(t, x, y) d m(y)\right)\left(\int_{s_{\delta}(x)} f^{2}(t, x, y) d m(y)\right)^{-1}}{1+\left(\int_{x-S_{\delta}(x)} f(t, x, y) p(t, x, d y)\right)\left(\int_{s_{\delta}(x)} f(t, x, y) p(t, x, d y)\right)^{-1}} .
\end{gathered}
$$

By (2.5) the expression in the numerator is not less than $\left(1-M P_{x}[H(t)]\right)^{2}$. Applying condition (D), with $A=\{x\}$ and $\eta=\delta$ to the second term in the denominator we find that for sufficiently small $t$ it does not exceed $N \cdot p\left(t, x, X-S_{\delta}(x)\right) p\left(t, x, S_{\delta}(x)\right)^{-1}$ where $N$ is a fixed positive number. The right continuity of the paths implies that this last expression approaches 0 as $t \rightarrow 0$, and since $P_{x}[H(t)] \rightarrow 0$ as $t \rightarrow 0$ Theorem 2.2 is established.

Let $N(\lambda)$ be the number of the eigenvalues $\{\lambda\}$ which do not exceed $\lambda$, that is $N(\lambda)=\sum_{\lambda_{j} \leq \lambda} 1$. We next prove the following theorem concerning the asymptotic behavior of $N(\lambda)$. 
Theorem 2.3. Suppose

$$
m(G)<\infty, \int_{G} f(t, x, x) d m(x)<\infty
$$

for all sufficiently small $t$, and

$$
\left[\int_{G} f^{2}(t, x, x) d m(x)\right]^{1 / 2}\left[\int_{G} f(t, x, x) d m(x)\right]^{-1}
$$

remains bounded as $t \rightarrow 0$. Then

$$
\int_{G} k(t, x, x) d m(x)\left(\int_{G} f(t, x, x) d m(x)\right)^{-1} \rightarrow 1 \quad \text { as } t \rightarrow 0 .
$$

If in addition $\int_{G} f(t, x, x) d m(x) \sim A t^{-\gamma}$ as $t \rightarrow 0$ for some $A$ and $\gamma>0$ then $N(\lambda) \sim A \lambda^{\gamma}(\Gamma(1+\gamma))^{-1}$ as $\lambda \rightarrow \infty$.

Proof. We have

$$
\begin{aligned}
& \frac{\int_{G} q(t, x, x) d m(x)}{\int_{G} f(t, x, x) d m(x)}=\frac{\int_{G} \frac{q(t, x, x)}{f(t, x, x)} f(t, x, x) d m(x)}{\int_{G} f(t, x, x) d m(x)} \\
& \leqq\left(\int_{G} \frac{q^{2}(t, x, x)}{f^{2}(t, x, x)} d m(x)\right)^{1 / 2} \frac{\left(\int_{G} f^{2}(t, x, x) d m(x)\right)^{1 / 2}}{\int_{G} f(t, x, x) d m(x)}
\end{aligned}
$$

$m(G)$ is finite, $q(t, x, x) f(t, x, x)^{-1}$ is bounded by 1 and by Theorem 2.2 approaches 0 as $t \rightarrow 0$ for all $x$ in $G$. The second factor in the last expression in (2.6) remains bounded as $t \rightarrow 0$, so

$$
\left(\int_{G} q(t, x, x) d m(x)\right)\left(\int_{G} f(t, x, x) d m(x)\right)^{-1} \rightarrow 0 \quad \text { as } t \rightarrow 0 .
$$

This yields the first assertion of Theorem 2.3. From the definition of $k$ it follows that

$$
\int_{G} k(t, x, x) d m(x)=\sum_{j=1}^{\infty} e^{-\lambda_{j} t}=\int_{0}^{\infty} e^{-\lambda t} d N(\lambda) .
$$

Thus by the first part of the theorem and the additional hypothesis of the second part we have

$$
\int_{0}^{\infty} e^{-\lambda t} d N(\lambda) \sim \int_{G} f(t, x, x) d m(x) \sim A t^{-\gamma}
$$

The conclusion of the theorem then follows by applying the Karamata tauberian theorem [6. p. 192]. 
3. Applications. In this section we apply the results of $\S 2$ to the symmetric stable processes and the Ornstein-Uhlenbeck processes on the real line. First consider the symmetric stable process of index $\alpha$ $(0<\alpha \leqq 2)$. Here $X=R^{1}, m$ is Lebesgue measure, and $f(t, x, y)=$ $g(t, x-y)$ where

$$
g(t, x)=\frac{1}{2 \pi} \int_{-\infty}^{\infty} e^{i x u} e^{-t|u|^{\alpha}} d u
$$

It is well known that the symmetric stable processes satisfy the conditions of $\S 2$ of $\mathrm{MO}$ and clearly $f$ is symmetric in $x$ and $y$. For each $t, f(t, x, y)$ is uniformly bounded so condition $(\mathrm{K})$ in $\S 6$ of $\mathrm{MO}$ is satisfied if $m(G)$ is finite (in particular if $\bar{G}$ is compact). We wish to verify condition (D) for the density $f$. To this end we state three lemmas.

Lemma 3.1. For each $t>0, g(t, x)$ decreases as $|x|$ increases.

LEMMA 3.2. Suppose $\rho$, a real valued function defined on $[0, \infty)$, has $N$ continuous derivatives and that $\varphi, \varphi^{(1)}, \cdots, \phi^{(N)}$ are all absolutely integrable on $[0, \infty)$. Suppose that for each $n \leqq N-1, \varphi^{(n)}(u) \rightarrow 0$ as $u \rightarrow \infty$. Then if $0<\lambda<1$ we have

$$
\begin{aligned}
& \int_{0}^{\infty} u^{\lambda-1} \varphi(u) \cos b u d u \\
=- & \sum_{n=0}^{N-1} \frac{\Gamma(n+\lambda)}{n !} \cos \left(\frac{\pi}{2}(n+\lambda-2)\right) \rho^{(n)}(0) b^{-n-\lambda}+O\left(b^{-N}\right) \text { as } b \rightarrow \infty .
\end{aligned}
$$

Lemma 3.3. For each $x \neq 0, g(t, x)$ is an increasing function of $t$ in the domain $0<t<B_{a}|x|^{x}$ where $B_{a}$ is a positive constant independent of $x$.

Lemma 3.1 is reasonably well known and a proof may be found in [7, Th. 11.8, p. 32]. Lemma 3.2 is a trivial modification of a theorem of Erdélyi [3, p. 48], to which we refer the reader. Lemma 3.3 is doubtless well known, but we are unable to find an explicit reference to it in the literature and so we give a proof.

Proof of Lemma 3.3. We fix $x \neq 0$ and look at the derivative $d g / d t=-(\pi)^{-1} \int_{0}^{\infty}(\cos x u) u e^{\alpha-t u^{\alpha}} d u$. Making the change of variable $t u^{\alpha}=y^{\alpha}$ we obtain

$$
\frac{d g}{d t}=-\frac{1}{\pi} t^{-1-1 / \alpha} h_{\alpha}(b)
$$

where $b=|x| t^{-1 / \alpha}$ and

$$
h_{a}(b)=\int_{0}^{\infty} y^{\alpha} e^{-y^{\alpha}} \cos b y d y .
$$


If $0<\alpha<1$ we apply Lemma 3.2 with $N=2, \lambda=\alpha$, and $\phi(y)=y e^{-y^{\alpha}}$. $\varphi$ clearly satisfies the assumptions of Lemma 3.2 and $\mathcal{P}(0)=0, \mathcal{P}^{\prime}(0)=1$ so we obtain

$$
\begin{aligned}
h_{\alpha}(b) & =-I^{\prime}(1+\alpha) \cos \left[\frac{\pi}{2}(\alpha-1)\right] b^{-1-x}+O\left(b^{-2}\right) & \\
& =-A(\alpha) b^{-1-\alpha}+O\left(b^{-2}\right) & \text { as } b \rightarrow \infty
\end{aligned}
$$

where $A(\alpha)=I^{\prime}(1+\alpha) \cos \left[\frac{\pi}{2}(\alpha-1)\right]>0$. If $1<\alpha<2$ we take $N=3$, $\lambda=\alpha-1$, and $\varphi(y)=y^{2} e^{-y^{\alpha}}$ and obtain

$$
h_{\alpha}(b)=-A(\alpha) b^{-1-\alpha}+O\left(b^{-3}\right)
$$

as $b \rightarrow \infty$.

If $0<\alpha<1$ then (3.4) implies that there are constants $M_{\alpha}$ and $b_{\alpha}$ such that $\left|h_{x}(b)+A(\alpha) b^{-1-\alpha}\right| \leqq M_{\alpha} b^{-2}$ if $b>b_{\alpha}$. Thus

$$
\left.\left.\left|\frac{d g}{d t}-\frac{A(\alpha)}{\pi}\right| x\right|^{-1-\alpha}\left|\leqq M_{\alpha}^{\prime}\right| x\right|^{-2} t^{-1+1 / \alpha}
$$

provided $|x| t^{-1 / \alpha}>b_{\alpha}$ or equivalently $0<t<b_{\alpha}^{\prime}|x|^{\prime \prime}$. Then $d g / d t$ will be positive if $M_{\alpha}^{\prime}|x|^{-2} t^{-1+1 / \alpha}<A(\alpha) \pi^{-1}|x|^{-1-\alpha}$ or equivalently if $0<t<M_{\alpha}^{\prime \prime}|x|^{\alpha}$. Thus if we take $B_{\alpha}=\min \left(b_{\alpha}^{\prime}, M_{\alpha}^{\prime \prime}\right)$ Lemma 3.3 is established for $0<\alpha<1$. If $1<\alpha<2$ a similar analysis beginning with (3.5) yields the desired result. Finally $g(t, x)=\pi^{-1} t\left(t^{2}+x^{2}\right)^{-1}$ if $\alpha=1$ and

$$
g(t, x)=(2 \sqrt{\pi t})^{-1} \exp \left(-x^{2} / 4 t\right)
$$

if $\alpha=2$ and the conclusion of the lemma is easily verified in these cases.

Now to verify condition (D) let $A$, a compact subset of $R^{1}$, and $\eta>0$ be given. If $t_{0}\left\langle B_{a} \eta^{r}\right.$ where $B_{x}$ is the constant of Lemma 3.3, if $|x-y|>\gamma$ and if $0<\sigma<t<t_{0}$ then $f(\sigma, x, y)=g(\sigma, x-y) \leqq g(t, x-y)$, and if $|x-z| \leqq \eta$ then

$$
\frac{f(\sigma, x, y)}{f(t, x, z)}=\frac{g(\sigma, x-y)}{g(t, x-z)} \leqq \frac{g(t, x-y)}{g(t, x-z)} \leqq 1
$$

the last inequality being a consequence of Lemma 3.1. In this case these estimates do not depend on $x$ being in $A$.

Since

$$
f(t, x, x)=g(t, 0)=(\pi)^{-1} \int_{0}^{\infty} e^{-t u^{x}} d u=(\alpha \pi)^{-1} t^{-1 / v} \Gamma(1 / \alpha),
$$

if $m(G)<\infty$ then the conditions of Theorem (2.3) are satisfied and we have 


$$
N(\lambda) \sim \frac{\lambda^{1 / \alpha}}{\pi} m(G)
$$

This is the asymptotic distribution of the eigenvalues for the symmetric stable process of index $\alpha$ on an open set $G$ of finite Lebesgue measure with $V$ bounded. This should be compared with the results of Kac [5]. (Kac's $V$ is different from ours. His $V \equiv 1$ yields our results with our $V \equiv 0$.)

Next we turn to the Ornstein-Uhlenbeck processes. It is well known [1] that these processes satisfy the conditions of $\S 2$ of MO (in fact the paths can be taken to be continuous.) The transition density relative to Lebesgue measure of the $0-U$ process with parameter $\beta>0$ is given by

$$
\frac{1}{\sqrt{2 \pi\left(1-\rho^{2}\right)}} \exp \left[-\frac{1}{2} \frac{(y-\rho x)^{2}}{1-\rho^{2}}\right]
$$

where $\rho=\rho(t)=e^{-\beta t}, \beta>0, t>0$. This density is not symmetric, but if we introduce the measure $m$ defined by $d m(y)=e^{-y^{2} / 2} d y$ then the transition density relative to $m$ is

$$
f(t, x, y)=\frac{1}{\sqrt{2 \pi\left(1-\rho^{2}\right)}} \exp \left[-\frac{1}{2} \frac{\rho^{2} x^{2}-2 \rho x y+\rho^{2} y^{2}}{1-\rho^{2}}\right]
$$

which is symmetric. We now verify condition (D) for this density. Let the compact set $A$ and number $\eta$ be given. Then

$$
\begin{gathered}
\frac{f(\sigma, x, y)}{f(t, x, z)} \\
=\frac{\left(1-\rho^{2}\right)^{-1 / 2}}{\left(1-\theta^{2}\right)^{-1 / 2}} \frac{\exp \left[-\frac{1}{2} \frac{(y-x)^{2}}{1-\rho^{2}}\right] \exp \left[-\frac{x y}{1+\rho}\right] \exp \left[\frac{x^{2}}{2}\right] \exp \left[\frac{y^{2}}{2}\right]}{\exp \left[-\frac{1}{2} \frac{(z-x)^{2}}{1-\theta^{2}}\right] \exp \left[-\frac{x z}{1+\theta}\right] \exp \left[\frac{x^{2}}{2}\right] \exp \left[\frac{z^{2}}{2}\right]}
\end{gathered}
$$

where $\rho=e^{-\beta \sigma}$ and $\theta=e^{-\beta t}$. The fourth factors in the numerator and denominator cancel. If we consider only $x, y$, and $z$ such that $x$ is in $A,|x-z|<\eta$ and $|y-x| \geqq \eta$ then the third and fifth factors in the denominator are bounded away from 0 and the second factor is no smaller than $\exp \left[-\frac{1}{2} \frac{\eta^{2}}{1-\theta^{2}}\right]$. Thus there exists a positive constant $N_{1}$ such that

$$
\begin{aligned}
& \frac{f(\sigma, x, y)}{f(t, x, z)} \\
\leqq & N_{1} \frac{\left(1-\rho^{2}\right)^{-1 / 2} \exp \left[-\frac{1(y-x)^{2}}{2} 1-\rho^{2}\right] \exp \left[-\frac{x y}{1+\rho}\right] \exp \left[\frac{y^{2}}{2}\right]}{\exp \left[-\frac{1}{2} \frac{\eta^{2}}{1-\theta^{2}}\right]}
\end{aligned}
$$


The product of the exponentials in the numerator is precisely

$$
\exp \left[-\frac{1(\rho y-x)^{2}}{21-\rho^{2}}\right]
$$

If $|y|>2 \max _{x \in A}|x|+2 \eta$ and $\rho>1 / 2$ then $(\rho y-x)^{2}>\gamma^{2}$. But for any other $y$ such that $|x-y| \geqq \eta$, the second and third exponentials in the numerator of (3.10) are uniformly bounded while the first exponential does not exceed $\exp \left[-\frac{1}{21-\rho^{2}}\right]$. Thus if $t_{0}$ is such that $e^{-\beta t_{0}}>1 / 2$, then for $\sigma<t \leqq t_{0}, x$ in $A,|x-y| \geqq \eta$, and $|x-z|<\eta$ we have

$$
\frac{f(\sigma, x, y)}{f(t, x, y)}<N_{2} \frac{\left(1-\rho^{2}\right)^{-1 / 2} \exp \left[-\frac{1}{21-\eta^{2}}\right]}{\left(1-\theta^{2}\right)^{-1 / 2} \exp \left[-\frac{1}{21-\theta^{2}}\right]}
$$

where $N_{2}$ is a positive constant. The right side of (3.11) is easily seen to be uniformly bounded for $0<\sigma<t \leqq t_{0}$ and thus condition (D) is verified.

For this density $f(t, x, x)=b(t) \exp \left(\rho x^{2} / 1+\rho\right)$ where $b(t)=\left[2 \pi\left(1-\rho^{2}\right)\right]^{-1 / 2}$ and $\rho=\rho(t)=e^{-\beta t}$. One verifies easily that if $\mu(G)<\infty$, where $\mu$ denotes Lebesgue measure, then condition $(K)$ as well as all the hypotheses of Theorem 2.3 are satisfied. In particular since $\rho$ increases to 1 as, $t \rightarrow 0$ we have

$$
\begin{aligned}
\int_{G} f(t, x, x) d m(x) & =b(t) \int_{G} e^{\left(\rho x^{2} / 1+\rho\right)} e^{-\left(x^{2} / 2\right)} d x & \\
& \sim b(t) \mu(G) \sim \frac{\mu(G)}{2 \sqrt{\beta \pi}} t^{-1 / 2} & \text { as } t \rightarrow 0 .
\end{aligned}
$$

So applying Theorem 2.3 we obtain for the $0-U$ process with parameter $\beta$

$$
N(\lambda) \sim \frac{\mu(G) \lambda^{1 / 2}}{\pi \sqrt{\beta}}
$$

If $G$ is the open interval $(a, b)$ then the infinitesimal generator $\Omega_{G}^{\prime}$ is given by the differential operator $\Omega_{G}^{\prime} \varphi=\beta\left[\varphi^{\prime \prime}+(x \varphi)^{\prime}\right]-V \varphi$ on an appropriate domain in $L_{2}[G, m]$ subject to the boundary conditions $\varphi(a)=$ $\phi(b)=0$. If $\beta=1$ notice that (3.12) reduces to (3.6) with $\alpha=2$. If $\alpha=2$ in (3.6) the corresponding infinitesimal generator is given by $\varphi^{\prime \prime}-V \rho$ on an appropriate domain with the same boundary conditions. Thus the term $(x \varphi)^{\prime}$ does not affect the asymptotic distribution of the eigenvalues, which is certainly what one would expect. The $\lambda_{\text {, }}$ are the eigenvalues of $-\Omega_{G}^{\prime}$ in each case. See Theorem 6.3 of MO. 


\section{REFERENCES}

1. J. L. Doob, The Brownian movement and stochastic equations, Ann. Math. 43. (1942), 351-369.

2. J. L. Doob, Stochastic Processes, New York 1953.

3. A. Erdélyi, Asymptotic Expansions, New York (Dover) 1956.

4. M. Kac, On some connections between probability theory and differential and integral equations, Proc. Second Berk. Symp. on Math. Stat. and Prob. (1951), 189-215.

5. M. Kac., Distribution of eigenvalues of ceriain integral operaiors, Mich. Math. J. 3 (1955-56), 141-148.

6. D. Widder, The Laplace Transform, Princeton 1949.

7. A. Wintner, Asymptotic Distributions and Infinite Convolutions, Edwards Brothers, Ann Arbor, Michigan, 1938.

UNIVERSITY OF WASHINGTON 


\section{PACIFIC JOURNAL OF MATHEMATICS}

\section{EDITORS}

\section{David Gilbarg}

Stanford University

Stanford, California

\section{R. A. Beaumont}

University of Washington

Seattle 5 , Washington

\author{
A. L. Whiteman
}

University of Southern California Los Angeles 7, California

L. J. Paige

University of California

Los Angeles 24, California

\author{
E. F. BECKENBACH \\ C. E. BURGESS \\ E. HEWITT \\ A. HORN
}

\author{
V. GANAPATHY IYER \\ R. D. JAMES \\ M. S. KNEBELMAN \\ L. NACHBIN
}

ASSOCIATE EDITORS
I. NIVEN

T. G. OSTROM

H. L. ROYDEN

M. M. SCHIFFER
E. G. STRAUS

G. SZEKERES

F. WOLF

K. YOSIDA

\section{SUPPORTING INSTITUTIONS}

\author{
UNIVERSITY OF BRITISH COLUMBIA \\ CALIFORNIA INSTITUTE OF TECHNOLOGY \\ UNIVERSITY OF CALIFORNIA \\ MONTANA STATE UNIVERSITY \\ UNIVERSITY OF NEVADA \\ OREGON STATE COLLEGE \\ UNIVERSITY OF OREGON \\ OSAKA UNIVERSITY \\ UNIVERSITY OF SOUTHERN CALIFORNIA
}

\author{
STANFORD UNIVERSITY \\ UNIVERSITY OF TOKYO \\ UNIVERSITY OF UTAH \\ WASHINGTON STATE COLLEGE \\ UNIVERSITY OF WASHINGTON \\ * * * \\ AMERICAN MATHEMATICAL SOCIETY \\ CALIFORNIA RESEARCH CORPORATION \\ HUGHES AIRCRAFT COMPANY \\ SPACE TECHNOLOGY LABORATORIES
}

Mathematical papers intended for publication in the Pacific Journal of Mathematics should be typewritten (double spaced), and the author should keep a complete copy. Manuscripts may be sent to any one of the four editors. All other communications to the editors should be addressed to the managing editor, L. J. Paige at the University of California, Los Angeles 24, California.

50 reprints per author of each article are furnished free of charge; additional copies may be obtained at cost in multiples of 50 .

The Pacific Journal of Mathematics is published quarterly, in March, June, September, and December. The price per volume (4 numbers) is $\$ 12.00$; single issues, $\$ 3.50$. Back numbers are available. Special price to individual faculty members of supporting institutions and to individual members of the American Mathematical Society: $\$ 4.00$ per volume; single issues, $\$ 1.25$.

Subscriptions, orders for back numbers, and changes of address should be sent to Pacific Journal of Mathematics, 2120 Oxford Street, Berkeley 4, California.

Printed at Kokusai Bunken Insatsusha (International Academic Printing Co., Ltd.), No. 6, 2-chome, Fujimi-cho, Chiyoda-ku, Tokyo, Japan.

PUBLISHED BY PACIFIC JOURNAL OF MATHEMATICS, A NON-PROFIT CORPORATION

The Supporting Institutions listed above contribute to the cost of publication of this Journal, but they are not owners or publishers and have no responsibility for its content or policies. 


\section{Pacific Journal of Mathematics}

\section{Vol. 9, No. $2 \quad$ June, 1959}

Lee William Anderson, On the breadth and co-dimension of a topological lattice

Frank W. Anderson and Robert L. Blair, Characterizations of certain lattices

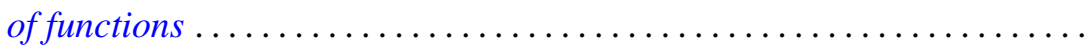

Donald Charles Benson, Extensions of a theorem of Loewner on integral

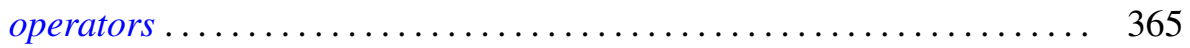

Errett Albert Bishop, A duality theorem for an arbitrary operator ........ 379

Robert McCallum Blumenthal and Ronald Kay Getoor, The asymptotic distribution of the eigenvalues for a class of Markov operators ........

Delmar L. Boyer and Elbert A. Walker, Almost locally pure Abelian

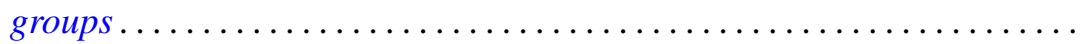

Paul Civin and Bertram Yood, Involutions on Banach algebras ........... Lincoln Kearney Durst, Exceptional real Lehmer sequences .... 415

Eldon Dyer and Allen Lowell Shields, Connectivity of topological

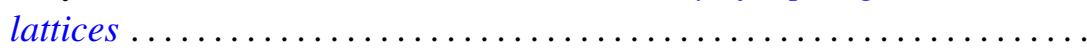

Ronald Kay Getoor, Markov operators and their associated

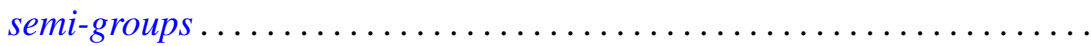

Bernard Greenspan, A bound for the orders of the components of a system of algebraic difference equations

Branko Grünbaum, On some covering and intersection properties in

Minkowski spaces ............................

Bruno Harris, Derivations of Jordan algebras ..............

Henry Berge Helson, Conjugate series in several variables.

Isidore Isaac Hirschman, Jr., A maximal problem in harmonic analysis.

II .

Alfred Horn and Robert Steinberg, Eigenvalues of the unitary part of a matrix

Edith Hirsch Luchins, On strictly semi-simple Banach algebras ...

William D. Munro, Some iterative methods for determining zeros of

functions of a complex variable...

John Rainwater, Spaces whose finest uniformity is metric .

William T. Reid, Variational aspects of generalized convex functions ....

A. Sade, Isomorphisme d'hypergroupoï des isotopes ...... . .

Isadore Manual Singer, The geometric interpretation of a special

connection . . .

Charles Andrew Swanson, Asymptotic perturbation series for characteristic

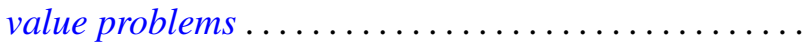

